\title{
Heat Transfer Characteristics of Ventilated Disc Brake Rotor with Diamond Pillars-A Review
}

\author{
Gorakh B. Kudal ${ }^{+*}$ and Mahesh R. Chopade ${ }^{\dagger}$ \\ †Department of Mechanical Engineering, MIT College of Engineering, Pune-411038, India
}

Accepted 03 March 2016, Available online 15 March 2016, Special Issue-4 (March 2016)

\begin{abstract}
Ventilated brake discs are used in modern vehicles such as passenger and racing cars as they require high speed braking system. The heat generated during braking operation is stored in brake disc and this heat is rejected to surrounding mainly by forced convection. Effective design of brake rotor is an essential to achieve proper cooling of braking system. The brake discs are available in various configurations such as radial type and pillared type brake rotors. It is found that thermal failure of brake disc is not only due to high temperature, but also due to high thermal stresses developed due to large temperature gradients within rotor passages. The temperature distribution within vane passage of radial vane rotors is not uniform and it leads to high thermal stresses. Pillared rotors are alternative to radial vane rotors as they have more uniform temperature distribution within rotor passages. Even though mass flow rate in case of pillared rotors is less than radial type rotors, the rate of heat transfer is almost same in both cases. This paper mainly focuses on understanding of air flow and heat transfer characteristics of diamond pillared rotor. Further, results of diamond pillared rotor are compared with other configurations of ventilated disc brake rotors.
\end{abstract}

Keywords: Pillared type brake rotors, thermal failure, thermal stresses, diamond pillared rotor

\section{Introduction}

Braking system is an essential component of vehicle for its safety purpose. Braking system is mainly used to control and limit the vehicle speed as per the requirements. Disc brakes are frictional brakes, which are used to convert kinetic energy of vehicle into thermal energy through friction between the brake pads and the rotor faces. Frictional brakes are mainly classified into disc brakes and drum brakes. There are numerous advantages of disc brakes over drum brakes such as disc brakes withstand in a very high temperature as compared to drum brakes; therefore they are generally used in high speed vehicles. Proper cooling of a vehicle braking system is one of its advantages. Long repetitive braking leads to temperature rise of various component of the vehicle brake system that reduces the performance of brake system. High temperatures can lead to overheating of brake fluid, seals and other components. The performance of disc brake increases by the rate at which heat is dissipated due to forced convection.

The temperature of rotor may reach very high temperatures during braking operation. Therefore, the disc brake must be able to dissipate the heat as fast as possible to ensure higher life expectancy. The brake

*Corresponding author: Gorakh B. Kudal discs are solid and ventilated type. The ventilated discs are mainly used to improve cooling performance and its operation is similar as centrifugal impeller. Ventilated disc brake rotors are preferred over solid rotors due to increase in surface area of heat transfer and reduction in mass of disc. The flow and heat transfer characteristics of ventilated disc brake rotors depend on various factors such as geometry of disc, air flow rate through rotor passages, air turbulence etc. The brake rotors are mainly divided into radial type and pillared type brake rotor.

The radial type brake rotors are easy to manufacture as compared to pillared type rotors. The material distribution is more uniform in case of pillared rotors, which provides resistance to crack propagation. It is found that thermal failure of brake rotor is not only due to high temperatures, but also due to high thermal stresses developed due to large temperature gradients.

The pressure, velocity and temperature distribution within vane passages of straight radial vane configuration rotors is not uniform, which leads to high thermal stresses. Heat transfer from the rotor passages is symmetrical in case of pillared rotors. Hence, for modern high-speed vehicles, ventilated pillared rotors may be more appropriate as compared with straight radial vane configuration rotors. To improve the performance of a vented brake rotor, an understanding 
of air flow and heat transfer phenomena is very important. The flow through passages of the ventilated disc brake rotor is highly complex in nature and it can be better understood by using computational fluid dynamics (CFD). Numerical modeling can predict flow and heat transfer characteristics within passages of brake rotor and it can be used to improve cooling performance of brake rotor.

\section{Ventilated Disc Brake Rotors}

Brake rotor is one of the important component of a disc brake system. During braking operation, large amount of heat is generated due to friction between stationary pads and rotating rotor faces. This heat must be rejected to atmosphere to avoid overheating and thermal distortion of brake rotor. The brake rotors are available in various designs. In earlier days solid disc brake rotors manufactured from cast iron were used. After development in automobile technology, there was a need to develop a new rotor which could provide better cooling. This need is fulfilled by ventilated disc brake rotors which have vanes joined together by the braking surfaces called the inboard and the outboard surface. They help in better heat dissipation from the rotor due to air flow in between the vanes and also due to increase in the area available to heat transfer. The ventilated disc brake rotor is shown in Fig. 1.

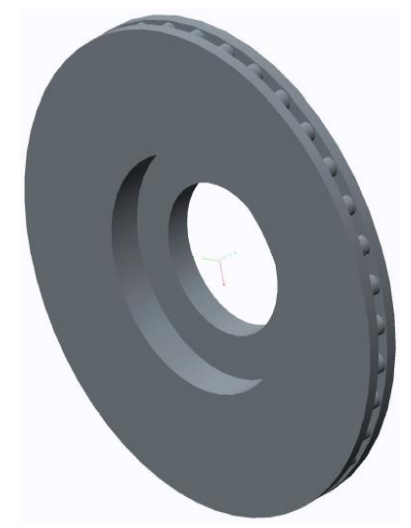

Fig.1: Ventilated Disc Brake Rotor

The operation of ventilated disc brake rotor is similar as centrifugal impeller in which air is passed in radial direction through vane passages due to action of centrifugal force. Ventilated disc brake rotor improves the convective cooling by circulating air through passages separating the braking surfaces. For years, ventilated brake rotors have been used for their advantages such as weight savings and increase in convective heat transfer area. However, the amount of additional cooling due to this internal air flow is not well defined and depends on the individual brake rotor's geometry and the cooling air flow conditions around the brake assembly.

There are different types of brake rotor configurations depending on the application of the vehicle. Some rotor configurations may be more effective than others, but they all help to serve one purpose - to provide even better cooling and braking performance. Four main types of brake rotor configurations are shown below in Fig. 2. First two rotor configurations are vane type known as straight radial vane (SRV) rotor and tapered radial vane (TRV) rotor. Tapered radial vane (TRV) rotor is modification of SRV rotor in which vanes are tapered in radially outward direction. Next two rotor configurations are pillared type known as diamond pillared (DP) and circular pillared (CP) brake rotor. In this configuration instead of vanes, pillars are used. The pillars may be circular or diamond in shape. The pillars are arranged in staggered manner as shown in Fig. 2.

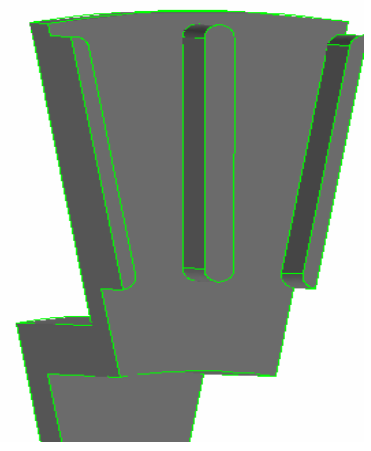

\section{Straight Radial Vane (SRV)}

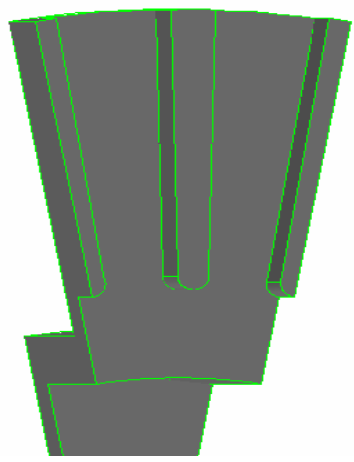

Tapered Radial Vane (TRV)

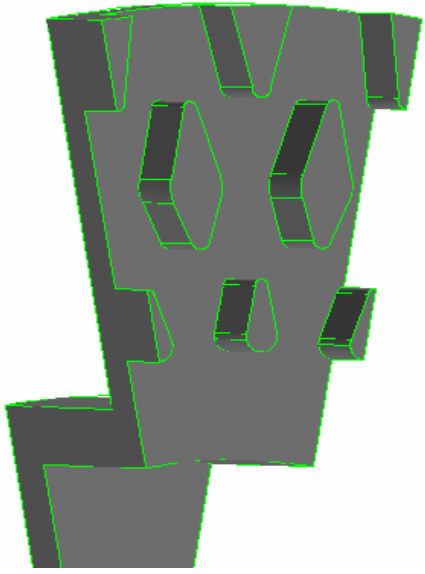

Diamond Pillar (DP) 


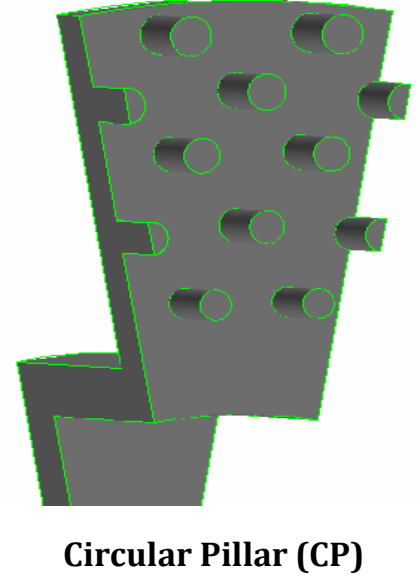

Fig. 2: Ventilated Disc Brake Rotor Configurations [1]

\section{Flow through Radial Vane Rotors}

The distribution of pressure, velocity and temperature of air at the vane passages of radial vane rotor is uneven. At the leading edge of passage, the pressure is more and it decreases as we go towards trailing edge. Also there is circulation region at the outlet of leading edge, which reduces air mass flow rate. This pressure distribution within passages of radial vane rotor is shown in Fig. 3. Due to uneven pressure distribution, high thermal stresses are generated across leading and trailing edge of passage, which leads to thermal failure of brake rotor. This non uniform temperature distribution promotes cracks, hotspots on rotor surfaces. Generally hot spots are generated due to very high temperature, $>700{ }^{\circ} \mathrm{C}$, concentrated at smaller region.

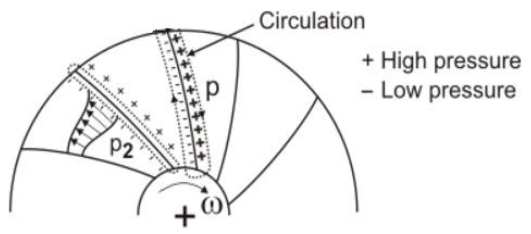

Fig. 3: Uneven Distribution of Pressure in Rotor Passages of Radial Vane Rotor

Compare to radial vane rotors, pillared rotors have more uniform temperature distribution within rotor passages, therefore they can sustain in a higher temperature. Due to this advantage the life of pillared rotor is more as compare to radial vane rotor and they can be suitable for high speed vehicles. The material distribution within rotor passages of pillared rotor is uniform, which provides better cracking resistance. Also due to arrangement of pillars air turbulence in passage also increases, which provides more heat dissipation characteristics.

\section{Comparison of Diamond Pillars with other Rotor Configurations}

Reddy S. et al. 2008 has performed CFD analysis on various ventilated rotor configurations as shown in
Fig.4 below. The figure shows the non-dimensional relative velocity vectors at the mid-plane of the passages of different rotors at $1800 \mathrm{rpm}$. From Fig. 4, it is observed that the recirculation region at the leading edge is reduced by modifying vane shape from straight radial vane (SRV) to tapered radial vane (TRV). Therefore mass flow rate of air is increased by about $33 \%$ for tapered radial vane (TRV) over straight radial vane (SRV) at $1800 \mathrm{rpm}$. Also it is seen that velocity distribution for pillared type rotors is more uniform as compared to radial vane rotors.
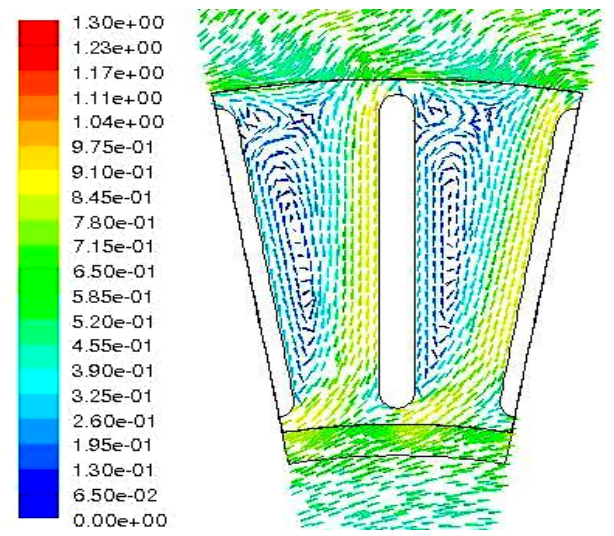

\section{Straight Radial Vane (SRV)}
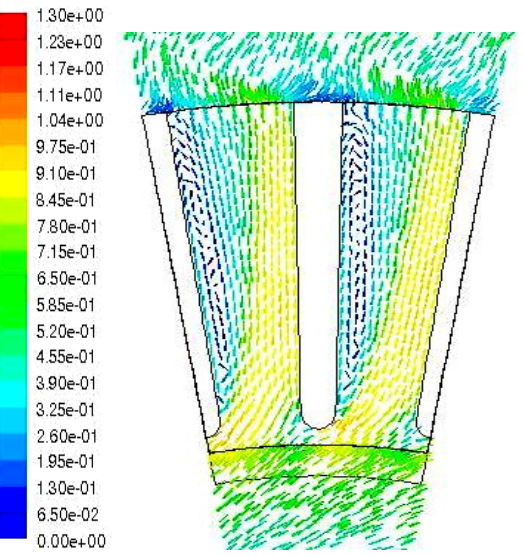

Tapered Radial Vane (TRV)

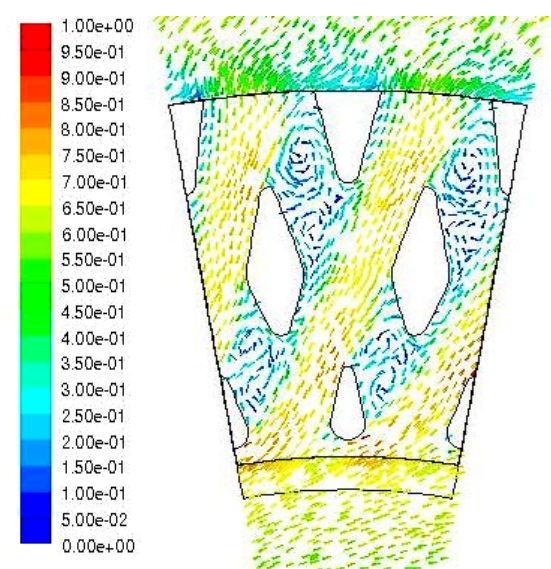

Diamond Pillar (DP) 

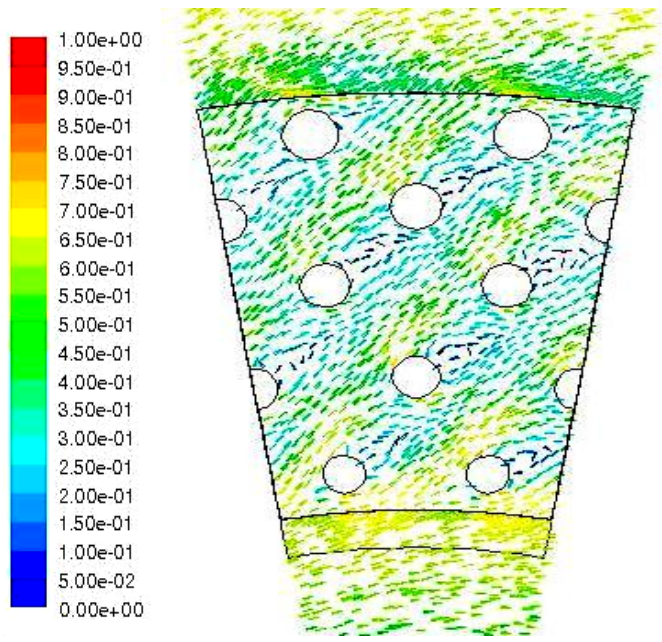

\section{Circular Pillar (CP)}

Fig. 4: Non-Dimensional Relative Velocity Vectors for Different Rotor Configurations

The cooling performance of brake rotor not only depends on mass flow rate, it mainly depends on passage geometry. The uniform distribution of mass flow rate in the passage is important for uniform heat dissipation from the rotor surfaces. Table 1 gives the mass flow rate, overall heat transfer coefficient and heat dissipation per passage through $20^{\circ}$ segments of different rotor configurations at rotational speed of $800 \mathrm{rpm}$ and at temperature of $700 \mathrm{~K}$.

Table 1: Comparison of Ventilated Disc Brake Rotor

\begin{tabular}{|c|c|c|c|}
\hline $\begin{array}{c}\text { Type of } \\
\text { Rotor }\end{array}$ & $\begin{array}{c}\text { Mass flow } \\
\text { rate (g/sec) }\end{array}$ & $\begin{array}{c}\text { Heat } \\
\text { Transfer } \\
\text { Coefficient } \\
(\mathrm{W} / \mathrm{m} 2 \mathrm{~K})\end{array}$ & $\begin{array}{c}\text { Heat } \\
\text { Dissipation } \\
\text { (W) }\end{array}$ \\
\hline SRV & 1.4 & 32.81 & 217.12 \\
\hline TRV & 2.04 & 45.26 & 295.12 \\
\hline DP & 1.51 & 42.11 & 279.15 \\
\hline CP & 0.72 & 32.71 & 216.13 \\
\hline
\end{tabular}

From above table, it is seen that the TRV rotor shows higher mass flow rate and heat transfer coefficient than all other rotor configurations. We can also see from Table 1 that the mass flow rate through passages of tapered radial vane (TRV) rotor is approximately $26 \%$ greater than diamond pillar rotor. Although diamond pillar (DP) has a noticeably lower mass flow rate than tapered radial vane (TRV) rotor, the passage heat dissipation is approximately same for both configurations. From this it is concluded that overall heat dissipation of ventilated disc brake rotor is highly depend on geometrical design of brake rotor. The flow of air through passages of diamond pillar (DP) rotor is more uniform than tapered radial vane (TRV) rotor. Therefore it is to be expected that the thermal gradients within passages of diamond pillar (DP) rotor will be less and temperature distribution is more uniform as compare to tapered radial vane (TRV) rotor.

\section{Conclusions}

In the present study, the thermal characteristics of diamond pillar brake rotor are compared with other configurations of brake rotor. From this comparison study, the following conclusions are drawn:

1) Mass flow rate through passages of diamond pillar (DP) rotor is $26 \%$ lower than tapered radial vane (TRV) rotor. Although diamond pillar (DP) has a noticeably lower mass flow rate than TRV rotor, the passage heat dissipation is approximately same for both configurations.

2) The heat dissipation through passages of diamond pillar (DP) rotor is $30 \%$ more than circular pillar (CP) rotor; hence diamond pillar (DP) may be more appropriate than circular pillar $(\mathrm{CP})$ rotor.

3) Airflow through passages of diamond pillar (DP) rotor is more uniform than tapered radial vane (TRV) rotor. Therefore temperature distribution within passages of diamond pillar (DP) brake rotor is more symmetrical, which leads to lower temperature gradients across rotor surfaces.

4) Due to uniform and higher heat dissipation characteristics, the diamond pillar (DP) brake rotor may be more appropriate for modern highspeed automobiles.

5) The brake rotor design should not be fully based on achievable heat transfer rate; it should also be based on the uniform heat dissipation from rotor surfaces.

\section{References}

Reddy S., Mallikarjuna J., Ganesan V., Flow and Heat Transfer Analysis of a Ventilated Disc Brake Rotor Using CFD, SAE Technical Paper 2008-01-0822, 2008.

Lisa Wallis et al., Air Flow and Heat Transfer in Ventilated Disc Brake Rotors with Diamond and Tear-Drop Pillars, Numerical Heat Transfer, Part A, 41:643-655, 2002.

Thundil Karuppa Raj Rajagopal et al., Numerical Investigation of Fluid Flow and Heat Transfer Characteristics on the Aerodynamics of Ventilated Disc Brake Rotor using CFD, Thermal Science: Year 2014, Vol. 18, No. 2, pp. 667-675.

Reddy S., Mallikarjuna J., Ganesan V., Effect of Vane Shape on Flow and Heat Transfer Characteristics of a Brake Disc, Proceedings of the 3rd BSME-ASME International conference on Thermal Engineering 20-22 December 2006, Dhaka, Bangladesh

Parish D., MacManus D. G., Aerodynamic Investigations of Ventilated Brake Discs, Proceedings of Institution of Mechanical Engineers, Part D: Journal of Automobile Engineering, 219, p.471-486, 2005.

Carlos Hannover Galindo-Lopez, Thesis on Optimization of Convective Heat Dissipation from Ventilated Brake Discs, May 2009.

Gautam Pulugundla, CFD Design Analysis of Ventilated Disc Brakes, May 2008

Yahya S. M., Turbines compressors and fans, Tata McGraw-Hill Education, 2010 